\title{
MULTIPLIERS AND ASYMPTOTIC BEHAVIOUR OF THE FOURIER ALGEBRA OF NONAMENABLE GROUPS
}

\author{
CLAUDIO NEBBIA 1
}

\begin{abstract}
Let $G$ be a locally compact group and $A(G)$ the algebra of matrix coefficients of the regular representation. We prove that $G$ is amenable if and only if there exist functions $u \in A(G)$ which vanish at infinity at any arbitrarily slow rate. The "only if" part of the result was essentially known. With the additional hypothesis that $G$ be discrete, we deduce that $G$ is amenable if and only if every multiplier of the algebra $A(G)$ is a linear combination of positive definite functions. Again, the "only if" part of this result was known.
\end{abstract}

1. Introduction. Let $G$ be a locally compact group; let $C_{0}(G)$ be the algebra of continuous complex-valued functions on $G$ which vanish at infinity. We use the definitions and the terminology of [3]. We let $B(G)$ be the Fourier-Stieltjes algebra consisting of all matrix coefficients of unitary representations of $G$, and $A(G)$ the Fourier algebra consisting of matrix coefficients of the regular representation. Regarded as the dual space of $C^{*}(G)$ (completion of $L^{1}(G)$ in the minimal regular norm), $B(G)$ is a Banach algebra under pointwise multiplication and $A(G)$ is a closed ideal in $B(G)$. We let $V N(G)$ be the von Neumann algebra of the regular representation $\lambda$ of $G$, which is the dual space of $A(G)$. It is known and easy to prove that if $G$ is amenable there exist functions in $A(G)$ which vanish at infinity at arbitrarily slow rates. More specifically, if $G$ is amenable, for every $f \in C_{0}(G)$, there exist $u \in A(G)$ and $g \in C_{0}(G)$ such that $f(x)=u(x) g(x)$. This means of course that $f(x)=o(u(x))$ as $x \rightarrow \infty$. We show that this property is characteristic of amenable groups. Theorem 1 contains this result and other equivalent characterizations of amenability.

Hence if $G$ is nonamenable, the coefficients of the regular representation must satisfy some condition of decrease at infinity. For particular nonamenable groups specific significant conditions are known; for instance, if $G$ is a semisimple Lie group with finite center, the Kunze-Stein phenomenon [2] implies that $A(G) \subset$ $\cap_{p>2} L^{p}(G)$, and if $G$ is a free group with at least two generators, a coefficient $u(x)$ of the regular representation must satisfy the condition $\left\{\Sigma_{|x|-n}|u(x)|^{2}\right\}^{1 / 2}=O(n)$ where $|x|$ denotes the length of the reduced word $x[9$, Theorem 3.1, p. 291].

Received by the editors December 4, 1980.

1980 Mathematics Subject Classification. Primary 43A07, 43A22.

Key words and phrases. Locally compact group, amenable group, multiplier of the Fourier algebra, Fourier-Stieltjes algebra, asymptotic behaviour of coefficients of the regular representation.

${ }^{1}$ The contents of this paper are part of the author's thesis for the Laurea in Mathematics at the University of Rome written under the supervision of Professor A. Figà-Talamanca.

This research is supported by "Istituto Nazionale di Alta Matematica F. Severi". 0002-9939/81/0000-1090/\$02.50 
Our result shows that these phenomena, which we could call "generalized Kunze-Stein phenomena," are characteristic of nonamenable groups. For the case of a discrete nonamenable group $G$, we use the result above to prove that there exist multipliers of $A(G)$ which are not in $B(G)$. This fact was known for groups containing a free group with two generators [7].

2. The general case. Recall that there is a natural module action of $A(G)$ on $V N(G)$ defined by

$$
\langle u \cdot T, v\rangle=\langle T, u v\rangle,
$$

for $u, v$ in $A(G)$ and $T$ in $V N(G)$. This allows us to introduce the following definition.

Defintion 1. A bounded linear operator $\Phi$ of $C_{0}(G)$ into $V N(G)$ is called a multiplier of $C_{0}(G)$ into $V N(G)$ if, for every $f$ in $C_{0}(G)$ and every $u$ in $A(G)$,

$$
\Phi(u f)=u \cdot \Phi(f) \text {. }
$$

Let $\mathscr{T}\left(C_{0}, V N\right)$ be the space of multipliers of $C_{0}(G)$ into $V N(G)$. It is not difficult to see that $\mathscr{R}\left(C_{0}, V N\right)$ can be identified with a subspace of $V N(G)$. It consists precisely of those elements $T$ in $V N(G)$ such that $\|T \mid\|<\infty$ where

$$
\|T\|=\sup \left\{\|u \cdot T\|_{V N}: u \in A(G),\|u\|_{\infty}<1\right\} .
$$

The norm $\||\cdot| \mid$ is exactly the operator norm of $\mathscr{T}\left(C_{0}, V N\right)$.

Let $M(G)$ be the space of bounded regular measures; it is clear that, for every $\mu$ in $M(G)$ and every $u$ in $A(G), u \mu \in M(G)$ where $d(u \mu)=u d \mu$.

Definition 2. A bounded linear operator $\Gamma$ of $A(G)$ into $M(G)$ is called a multiplier of $A(G)$ into $M(G)$ if, for every $u$ and $v$ in $A(G)$,

$$
\Gamma(u v)=u \Gamma(v) \text {. }
$$

Let $\mathscr{T}(A, M)$ be the Banach space of multipliers of $A(G)$ into $M(G)$ with the operator norm. Let $\mathfrak{T}\left(A, L^{1}\right)$ be the subspace of $\mathscr{T}(A, M)$ which consists of those elements $\Gamma$ such that $\Gamma(u) \in L^{1}(G)$ for every $u$ in $A(G)$.

It is clear that for every $f$ in $L^{1}(G)$ the operator $\Gamma(u)=u f$ is a multiplier of $A(G)$ into $L^{1}(G)$.

It is easy to see that the space $\mathfrak{R}(A, M)$ is isometrically isomorphic to the space $\mathscr{T}\left(C_{0}, V N\right)$, since $M(G)$ and $V N(G)$ are, respectively, the dual spaces of $C_{0}(G)$ and $A(G)$.

The isomorphism is given by the following formula:

$$
\langle\Phi(f), v\rangle=\langle f, \Gamma(v)\rangle
$$

for $\Gamma$ in $\Re(A, M), \Phi$ in $\Re\left(C_{0}, V N\right), f$ in $C_{0}(G)$ and $v$ in $A(G)$.

If $\mu \in M(G)$ then $\Gamma(u)=u \mu$, for $u$ in $A(G)$, is a multiplier of $A(G)$ into $M(G)$ and $\|\Gamma\| \mid<\|\mu\|$.

Moreover every multiplier is a Radon measure, generally unbounded. Indeed suppose that $K$ is a compact subset of $G$ and let $v_{K}$ be a function in $A(G)$ such that $v_{K}(x)=1$ for every $x$ in $K$. If $u$ is a function in $A(G)$ with support in $K$, then

$$
\begin{aligned}
|\langle T, u\rangle| & =\left|\left\langle T, u v_{K}\right\rangle\right|=\left|\left\langle u \cdot T, v_{K}\right\rangle\right| \\
& =\left|\left\langle u, \Gamma\left(v_{K}\right)\right\rangle\right|\left\langle\|u\|_{\infty}\|\Gamma \mid\| v_{K} \|_{A}\right.
\end{aligned}
$$

where $T$ is the element in $\mathscr{N}\left(C_{0}, V N\right)$ corresponding to $\Gamma$ in $\mathscr{N}(A, M)$. 
The Radon measures, generally, are unbounded, but we shall see that every multiplier is a bounded Radon measure if and only if $G$ is amenable.

For every multiplier $\Gamma$ in $\mathscr{N}(A, M)$ let $|\Gamma|$ be the absolute value of $\Gamma$ in the measure theoretic sense. Then also $|\Gamma|$ is a multiplier of $A(G)$ into $M(G)$ and $\||\Gamma|\|=|||\Gamma|||$.

This follows from the fact that, for every $u$ in $A(G)$ with compact support $K$,

$$
\left\langle u,|\Gamma|\left(v_{K}\right)\right\rangle=\left\langle u,\left|\Gamma\left(v_{K}\right)\right|\right\rangle \text { and }\|\Gamma(u)\|=\||\Gamma|(u)\| .
$$

Finally, we observe that if $S$ and $T$ are Radon measures such that $|S|<|T|$, then $\||| S|\||\leqslant||| T \mid\|$.

Hence if $T$ is a multiplier then also $S$ is a multiplier because the multipliers of $A(G)$ into $M(G)$ are exactly the Radon measures $T$ such that $\|T\|<\infty$.

Definition 3. Let $X$ be the subspace of $C_{0}(G)$ defined as follows:

$$
X=\left\{h: h(x)=\sum_{i=1}^{\infty} u_{i}(x) g_{i}(x), u_{i} \in A(G), g_{i} \in C_{0}(G), \sum_{i=1}^{\infty}\left\|u_{i}\right\|_{A}\left\|g_{i}\right\|_{\infty}<\infty\right\} \text {. }
$$

We define the norm:

$$
\|h\|_{X}=\inf \left\{\sum_{i=1}^{\infty}\left\|u_{i}\right\|_{A}\left\|g_{i}\right\|_{\infty}: \sum_{i=1}^{\infty} u_{i}(x) g_{i}(x)=h(x)\right\} .
$$

It is not difficult to show that, with the norm $\|\cdot\|_{X}$, the space $X$ is a Banach space. We can also prove the following lemma.

LEMMA 1. The space $\mathfrak{T}\left(C_{0}, V N\right)$ is isometrically isomorphic to the dual space of $X$; the duality is given by the following formula:

$$
\langle\Phi, h\rangle=\sum_{i=1}^{\infty}\left\langle\Phi\left(g_{i}\right), u_{i}\right\rangle
$$

for $h$ in $X, h=\Sigma_{i} u_{i} g_{i}$, and $\Phi$ in $\Re\left(C_{0}, V N\right)$.

Proof. We can apply the proof of [6, Theorem 1], observing that $C_{0}(G)$ always has an approximate identity, bounded in the supremum norm, which consists of elements of $A(G)$.

THEOREM 1. If $G$ is a locally compact group, the following are equivalent:

(a) $G$ is amenable.

(b) For every $f$ in $C_{0}(G)$ there exist $u$ in $A(G)$ and $g$ in $C_{0}(G)$ such that $u(x) g(x)=f(x)$.

(c) $\mathfrak{T}(A, M) \simeq \mathfrak{T}\left(C_{0}, V N\right)=M(G)$.

(d) $\mathfrak{N}\left(A, L^{1}\right)=L^{1}(G)$.

Proof. (a) implies (b). If $G$ is amenable there is a bounded approximate identity in $A(G)$ [13]. Since $C_{0}(G)$ is an $A(G)$-module, (b) follows from the factorization theorem for Banach modules [11, (32.22)]. If (b) holds, the space $X$ defined above is $C_{0}(G)$ and (c) follows from Lemma 1.

If (c) holds for every $\Gamma$ in $\Re\left(A, L^{1}\right)$ there exists a bounded measure $\mu$ in $M(G)$ such that $\Gamma(u)=u \mu$ and $u \mu \in L^{1}(G)$ for every $u$ in $A(G)$; hence, $\mu \in L^{1}(G)$ and (d) follows. We prove now that (d) implies (a). 
It is known [14] that $G$ is amenable if and only if $\|f\|_{1}=\|\lambda(f)\|$ for every nonnegative $f$ in $L^{1}(G)$. We observe that, for $f$ in $L^{1}(G)$ and $u$ in $A(G)$,

$$
\|u \cdot \lambda(f)\|=\|\lambda(u f)\|<\|u\|_{\infty}\|\lambda(|f|)\| \text {. }
$$

Indeed if $g \in L^{2}(G)$,

$$
\begin{aligned}
\|\lambda(u f) g\|_{2} & =\|u f * g\|_{2} \leqslant\||u f| *|g|\|_{2} \\
& <\|\| u\left\|_{\infty}|f| *|g|\right\|_{2}<\|u\|_{\infty}\|\lambda(|f|)\|\|g\|_{2} .
\end{aligned}
$$

This means that if $f>0$, then $\|u \cdot \lambda(f)\|<\|u\|_{\infty}\|\lambda(f)\|$ and therefore $\|\lambda(f)\|<$ $\|\lambda(f)\|$ for a nonnegative $f$ in $L^{1}(G)$.

If (d) holds, the norm $\|\cdot\|_{1}$ of $L^{1}(G)$ is equivalent to $\|\cdot\| \|$ (by the closed graph theorem). In particular, $\|f\|_{1}<k\|\| \lambda(f) \|$ for every $f$ in $L^{1}(G)$. Hence $\|f\|_{1}<$ $k\|\lambda(f)\|$ for every nonnegative $f$ in $L^{1}(G)$. But if $f>0$,

$$
\left\|f * f^{*}\right\|_{1}=\|f\|_{1}^{2} \text {. }
$$

This implies that $\|f\|_{1}<\sqrt{k}\|\lambda(f)\|$, which means $\|f\|_{1}<\|\lambda(f)\|$ and (a) follows from [14, Theorem 1].

In the following corollary we prove that, for nonamenable groups, the condition of decrease at infinity of the Fourier algebra is a summability condition with respect to a positive measure.

COROLlary. If $G$ is nonamenable there exists a positive Radon measure $\mu$ such that:

(a) $\mu(G)=+\infty$,

(b) $A(G) \subset L^{p}(\mu)$ for every $1<p \leqslant \infty$.

Proof. If $G$ is nonamenable there exists a multiplier $\Gamma$ of $A(G)$ into $L^{1}(G)$ which is not in $L^{1}(G)$. Then also $|\Gamma|$ is a multiplier of $A(G)$ into $L^{1}(G)$ which is not in $L^{1}(G)$. So $\mu=|\Gamma|$ is a positive Radon measure such that

$$
\mu(G)=+\infty \text { and } A(G) \subset L^{1}(\mu) .
$$

Finally $A(G) \subset L^{\infty}(\mu)$, obviously, and (b) follows.

REMARKS. (1) Observe that the statement (d) of Theorem 1 is true for every $1<p<\infty$. Indeed let $\pi\left(A, L^{p}\right)$ be the space of multipliers of $A(G)$ into $L^{p}(G)$ for $1<p<\infty$, i.e. the Banach space, with the operator norm, of the bounded linear operators $\Phi$ of $A(G)$ into $L^{p}(G)$, such that $\Phi(u v)=u \Phi(v)$ for every $u$ and $v$ in $A(G)$. So, if $G$ is amenable there is a bounded approximate identity in $A(G)$; hence, $\mathscr{T}\left(A, L^{p}\right)=L^{p}(G)$ for every $1<p<\infty$. Conversely, if $G$ is nonamenable and $1<p<\infty$, then there exists a multiplier $\Gamma$ of $A(G)$ into $L^{1}(G)$ which is not in $L^{1}(G)$. But if $K$ is a compact subset of $G$ then we can find a function $f$ such that $\Gamma(u)=f u$ for every $u$ in $A(G)$ whose support is in $K$; hence, $\Phi(u)=|f|^{1 / p_{u}}$ is a multiplier of $A(G)$ into $L^{p}(G)$ which is not in $L^{p}(G)$.

(2) The space of multipliers of $A(G)$ into $l^{1}(G)$ for discrete groups was studied by $M$. Bożejko in [1]; he proves that a discrete group $G$ is amenable if and only if $l^{1}(G)=l^{1}[G]$ where $l^{1}[G]$ is the closure, in $\mathscr{N}\left(A, l^{1}\right)$, of the space of functions with finite support. 
(3) Observe that, in the corollary, the measure $\mu$ is 2-admissible since $A(G) \subset$ $L^{1}(\mu)($ see $[14,8,5])$.

3. The discrete case. Let $G$ be a discrete group; then $M(G)=l^{1}(G)$ and $\mathfrak{T}\left(A, l^{1}\right)$, defined above, is exactly the space of all functions $\varphi$ on $G$ such that $\varphi u \in l^{1}(G)$ for every $u$ in $A(G)$.

Definition 4. If $X$ and $Y$ are two spaces of functions on $G$, let $\mathscr{T}(X, Y)$ be the space of the multipliers of $X$ into $Y$, i.e. the functions $\varphi$ on $G$ such that $\varphi g \in Y$ for every $g$ in $X$.

If $X$ and $Y$ are Banach spaces, $\mathscr{R}(X, Y)$ is a Banach space in the operator norm.

LEMMA 2. $\mathscr{T}\left(l^{\infty}, B\right)=l^{2}(G)$.

Proof. Let $\varphi$ be in $\Re\left(l^{\infty}, B\right)$ and let $F$ be a finite subset of $G$. Let $\varphi_{F}=\chi_{F} \varphi$, where $\chi_{F}$ is the characteristic function of the set $F$; then

$$
\left\|\varphi_{F}\right\|_{A}=\left\|\varphi \chi_{F}\right\|_{B}<\|\varphi\|\|\| \chi_{F}\left\|_{\infty}=\right\| \varphi\|\|
$$

where $\left\||\cdot \||\right.$ is the norm of $\operatorname{Tl}\left(l^{\infty}, B\right)$.

Define as in [15, Definition 4] the space $L^{2 s}, s=1,2, \ldots$, as the completion of the space of the finitely supported functions with the norm $\|f\|_{L^{25}}=$ $\left(\left[f * f^{*}\right]^{s}\left(1_{G}\right)\right)^{1 / 2 s}$, where the power is meant as a convolution power. Then the space $L^{2}$ is the same as $l^{2}$, and the proof of [15, Theorem 2] shows that there exists a function $u$ of modulus one on $F$ such that

$\left[15\right.$, p. 503]. In particular $\left\|u \varphi_{F}\right\|_{L^{4}}<2 \sqrt{2}\left\|\varphi_{F}\right\|_{l^{2}}$. But

$$
\begin{gathered}
\left\|u \varphi_{F}\right\|_{L^{2 s}} \leqslant 2 \sqrt{s}\left\|\varphi_{F}\right\|_{L^{2}}, \\
\|f\|_{L^{2}} \leqslant\|f\|_{A}^{1 / 3}\|f\|_{L^{4}}^{2 / 3} .
\end{gathered}
$$

(This inequality can be deduced from the corresponding inequality for ordinary $L^{p}$-spaces, where $L^{1}$ takes the place of $A(G)$, by using the fact that the trace $\operatorname{tr}(f)=f\left(1_{G}\right)$ acts as an ordinary integral on any commutative *-subalgebra of $V N(G)[12 ; 15$, Remark 4].)

We conclude that for some $u$ of modulus one,

$$
\begin{aligned}
\left\|\varphi_{F}\right\|_{l^{2}} & =\left\|u \varphi_{F}\right\|_{l^{2}} \leqslant\left\|u \varphi_{F}\right\|_{A}^{1 / 3}\left\|u \varphi_{F}\right\|_{L^{4}}^{2 / 3} \\
& \leqslant(2 \sqrt{2})^{2 / 3}\left\|u \varphi_{F}\right\|_{A}^{1 / 3}\left\|\varphi_{F}\right\|_{l^{2}}^{2 / 3}
\end{aligned}
$$

hence $\left\|\varphi_{F}\right\|_{l^{2}} \leqslant 8\left\|u \varphi_{F}\right\|_{A}=8\left\|u \varphi_{F}\right\|_{B} \leqslant 8\|\| \varphi \|$.

Therefore $\varphi \in l^{2}(G)$, as $F$ is arbitrary.

THEOREM 2. If $G$ is a discrete group, the following are equivalent:

(a) $G$ is amenable.

(b) $\mathfrak{R}(A, A)=B(G)$.

Proof. It is known that (a) implies (b) for locally compact groups [5, 10, 16]. We prove now that (b) implies (a).

If $G$ is nonamenable there exists a multiplier $f$ of $A(G)$ into $l^{1}(G)$ which is not in $l^{1}(G) ;$ it follows from Theorem 1 . 
Then $h=|f|^{1 / 2}$ is a multiplier of $A(G)$ into $l^{2}(G)$ which is not in $l^{2}(G)$, as is easily seen. From Lemma 2 it follows that there exists a function $\varphi$ in $l^{\infty}(G)$ such that $\varphi h$ is not in $B(G)$, but $\varphi h \in \mathfrak{N}\left(A, l^{2}\right) \subset \mathfrak{N}(A, A)$. Hence $B(G) \neq \mathscr{N}(A, A)$.

We thank the referee for his valuable suggestions.

\section{REFERENCES}

1. M. Bożejko, A new group algebra and lacunary sets in discrete noncommutative groups, Studia Math. (to appear).

2. M. Cowling, The Kunze-Stein phenomenon, Ann. of Math. (2) 107 (1978), 209-234.

3. P. Eymard, L'algèbre de Fourier d' un groupe localement compact, Bull. Soc. Math. France 92 (1964), $181-236$.

4. , Algèbres $A_{p}$ et convoluteurs de $L^{p}$ (Séminaire Bourbaki, 1969/70) Lecture Notes in Math., vol. 180, Springer-Verlag, Berlin and New York, 1971, pp. 55-72. , Initiation à la théorie des groupes moyennables (Séminaire Nancy-Strasbourg, 1973/75), Lecture Notes in Math., vol. 497, Springer-Verlag, Berlin and New York, 1975, pp. 89-107.

6. A. Figà-Talamanca, A remark on multipliers of the Fourier algebra of the free group, Boll. Un. Mat. Ital. A (5) 16 (1979), 577-581.

7. A. Figà-Talamanca and M. A. Picardello, Multiplicateurs de $A(G)$ qui ne sont pas dans $B(G), \mathrm{C}$. R. Acad. Sci. Paris Sér. A 277 (1973), 117-119.

8. J. E. Gilbert, Convolution operators on $L^{p}(G)$ and properties of locally compact groups, Pacific J. Math. 24 (1968), 257-268.

9. U. Haagerup, An example of a non nuclear $C^{*}$-algebra which has the metric approximation property, Invent. Math. 50 (1979), 279-293.

10. C. Herz, Une généralisation de la notion de transformée de Fourier-Stieltjes, Ann. Inst. Fourier (Grenoble) (3) 24 (1974), 145-157.

11. E. Hewitt and K. A. Ross, Abstract harmonic analysis, vol. II, Springer-Verlag, Berlin and New York, 1970.

12. R. Kunze, $L_{p}$ Fourier transforms on locally compact unimodular groups, Trans. Amer. Math. Soc. 89 (1958), 519-540.

13. H. Leptin, Sur l'algèbre de Fourier d'un groupe localement compact, C. R. Acad. Sci. Paris Sér. A 266 (1968), 1180-1182.

14. On locally compact groups with invariant means, Proc. Amer. Math. Soc. 19 (1968), 489-494.

15. M. A. Picardello, Lacunary sets in discrete noncommutative groups, Boll. Un. Mat. Ital. (4) 8 (1973), 494-508.

16. P. F. Renaud, Centralizers of the Fourier algebra of an amenable group, Proc. Amer. Math. Soc. 32 (1972), 539-542.

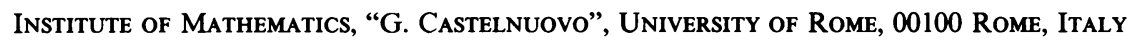

